\title{
Minocycline Superior to Chloroquine Phosphate as COVID-19 Treatment
}

\author{
Mosab Nouraldein Mohammed Hamad
}

Research Unit, Banoon Fertility Center, Sudan

\begin{tabular}{ll}
\hline DOI: $10.36348 /$ sjbr.2020.v05i03.006 & | Received: 07.03 .2020 | Accepted: 19.03 .2020 | Published: 30.03 .2020
\end{tabular}

*Corresponding author: Mosab Nouraldein Mohammed Hamad

\section{Abstract}

COVID-19 infection is the current challenge that faces the research community worldwide. Although chloroquine phosphate, an old drug for management of malaria, revealed to have clear value and tolerable safety against COVID-19 associated pneumonia in multicenter clinical trials conducted in China, we theorize that minocycline combined with suitable non-steroidal anti-inflammatory drug will give better result than Chloroquine phosphate monotherapy. Nouraldein theory needs invitro and Invivo experiments to confirm it.

Keywords: COVID-19 infection, Chloroquine phosphate, Minocycline, non-steroidal anti-inflammatory agent.

Copyright @ 2020: This is an open-access article distributed under the terms of the Creative Commons Attribution license which permits unrestricted use, distribution, and reproduction in any medium for non-commercial use (NonCommercial, or CC-BY-NC) provided the original author and source are credited.

\section{INTRODUCTION}

Coronaviruses are envelop, positive singlestranded large RNA viruses that attack humans, but also a broad range of animals. They were first reported in 1966 by Tyrell and Bynoe, who cultured the viruses from patients with common colds. According on their morphology as spherical virions with a center shell and surface projections similar to a solar corona, they were named coronaviruses (Latin: corona $=$ crown). Four subfamilies, namely alpha-, beta-, gamma- and delta coronaviruses be present. As alpha- and betacoronaviruses actually derive from bats, gamma- and delta-viruses derive from pigs and birds.

Covid-19 actually succeeded in building its transition from animals to humans on the Huanan seafood marketplace in Wuhan, China. The early clinical feature which allowed case recognition was pneumonia. More fresh accounts also explain gastrointestinal symptoms and asymptomatic infections, particularly among young kids [1].

On February 17, 2020, the State Council of China held a news briefing representing that chloroquine phosphate, an old medicine for cure of malaria, had established noticeable effectiveness and adequate safety in treating COVID-19 associated pneumonia in multicenter clinical trials carried out in China [2]. Chloroquine Phosphate is the phosphate salt of chloroquine, a quinoline compound with antimalarial and anti-inflammatory characteristics. Chloroquine is the most extensively used drug against malaria, excepting for those cases resulted by chloroquine resistant Plasmodium falciparum. Though the method of action is not fully understood, chloroquine is shown to slow down the parasitic enzyme heme polymerase that changes the toxic heme into non-toxic hemazoin, so resulting in the gathering of toxic heme within the parasite. Chloroquine may also disrupt the biosynthesis of nucleic acids [3].

Presently, there is no efficient vaccine or treatment for COVID-19 infection; hence, there is a significant need for other and useful drug choices. Minocycline; a tetracycline-class wide-spectrum antibiotic, employed initially to treat various bacterial infections, that is currently also employed for treating neurological problems. It is recognized to display antioxidant, anti-inflammatory, anti-apoptotic, and anticancer properties, which formulates it advantageous for the management of multiple sclerosis as well as Alzheimer's, Huntington's, and Parkinson's diseases. Furthermore, minocycline has been shown to be an effectual antiviral drug against many viral attacks including HIV infection [4].

Minocycline is approximately entirely absorbed after oral administration. Following a single $200 \mathrm{mg}$ dose, climax serum levels of about 2 to $3 \mu \mathrm{g} / \mathrm{ml}$ are reached in 2 hours in fit subjects and absorption is not noticeably prejudiced by the presence of food or milk. The absorption of minocycline may be unfavorably influenced by the concurrent administration of iron-containing haematinics and by antacids containing aluminium hydroxide, calcium and 
magnesium ions, all of which interfere with the absorption of tetracyclines and therefore, lower their in vivo antimicrobial action [5]. Because of the anticollagenase activity of minocycline initially recognized by Golub et al. and later confirmed by Greenwald et al. in human being rheumatoid tissue and synovial cultures, a lot of studies through the last four decades have alert on its influences in rheumatoid arthritis. In the adjuvant arthritis model of rheumatoid arthritis, tetracyclines decreased collagenase action in the inflamed tissue, though they did not show antiinflammatory effects in rats. When combined with nonsteroidal anti-inflammatory drugs in the similar study, tetracyclines had a synergistic outcome, as evidenced by a whole suppression of degradative enzyme action and a normalization of radiological bone damage [6]. Study dony by QINGHUA LIN1 et al showed that; minocycline was the most powerful for inhibiting the growth of cultured P. falciparum. It finally showed killing activity for mefloquine-resistant P. falciparum parasites as well as chloroquine- and pyrimethamineresistant parasites, while these antibiotics need a longer time for an emergence of the inhibitory outcome than other antimalarial drugs [7].

Study done by Swapnil S. Bawage et al. showed that; minocycline reduces the respiratory syncytial virus Infection-mediated cytopathic influence and prevents RSV infection [4].

\section{Nouraldein theory}

Minocycline combined with non-steroid antiinflammatory drug will be successful treatment for COVID-19 more than Chloroquine phosphate due to its ability to overcome Chloroquine resistant illnesses.

\section{RECOMMENDATIONS}

The theory needs extensive invitro and Invivo experiments to confirm it, and to start usage of combination therapy to cure COVID-19 patients.

\section{REFERENCES}

1. Velavan, T. P., \& Meyer, C. G. (2020). The COVID-19 epidemic. Trop Med Int Health, 25(3), 278-280.

2. Gao, J., Tian, Z., \& Yang, X. (2020). Breakthrough: Chloroquine phosphate has shown apparent efficacy in treatment of COVID-19 associated pneumonia in clinical studies. Bioscience trends.

3. Bawage, S. S., Tiwari, P. M., Pillai, S., Dennis, V. A., \& Singh, S. R. (2019). Antibiotic Minocycline Prevents Respiratory Syncytial Virus Infection. Viruses, 11(8), 739.

4. Brogden, R. N., Speight, T. M., \& Avery, G. S. (1975). Minocycline: a review of its antibacterial and pharmacokinetic properties and therapeutic use. Drugs, 9(4), 251-291.

5. Garrido- Mesa, N., Zarzuelo, A., \& Gálvez, J. (2013). Minocycline: far beyond an antibiotic. British journal of pharmacology, 169(2), 337-352.

6. LIN, Q., KATAKURA, K., OUE, M., KANO, S., \& SUZUKI, M. (2001). Effects of minocycline against mefloquine-, chloroquine-and pyrimethamine-resistant Plasmodium falciparum in vitro. Japanese Journal of Tropical Medicine and Hygiene, 29(4), 343-348.

7. Chloroquine phosphate, https://pubchem.ncbi.nlm.nih.gov/compound/Chlor oquine-phosphate. 